\title{
Cholesterol Lowering Potential of Lactobacillus brevis
}

\author{
Ma. Gladys B. Aquino ${ }^{1}$, Bernard U. Ebuen ${ }^{1}$, Cristina Widwidan", Gil P. Soriano ${ }^{2}$, \\ ${ }^{1}$ College of Medical Laboratory Science, Arellano University, Philippines \\ ${ }^{2}$ School of Medical Technology, Centro Escolar University, Philippines
}

\begin{abstract}
High cholesterol has been attributed to be a risk factor for cardiovascular diseases. Current measures include dietary changes, exercise and therapeutic agents that lower cholesterol levels. However, pharmacologic agents have significant side-effects and limitations. There has been an increasing interest in probiotics which can lower cholesterol. Lactobacillus has been reported to inhibit cholesterol synthesis and promote excretion of acidic sterol. In this study, Lactobacillus brevis was investigated for its cholesterol lowering effect. To evaluate the cholesterol assimilating property of Lactobacillus brevis, MRS broth with different levels of water-soluble cholesterol was added to culture media and the cholesterol concentration of supernatant broth and pellets were analysed after 24 hours incubation. The results of all MRS broth showed significant reduction of cholesterol both in the supernatant and pellet. This result provides an initial screening of Lactobacillus brevis for their efficacy as cholesterol-lowering therapeutics and demonstrates their potential use as cholesterol-lowering agents.
\end{abstract}

Keywords: cholesterol, assimilation, MRS broth, Lactobacillus, probiotic

\section{INTRODUCTION}

Hypercholesterolemia is a disease that has been prevailing worldwide and is characterized by elevated levels of cholesterol in the blood. It has been attributed to be a proven risk factor that may result in other forms of diseases like cardiovascular diseases (CVD) and cerebrovascular accidents (CVA). CVD is the leading cause of death globally which accounts for 17.7 million deaths globally in 2015 representing $31 \%$ of all global deaths [1]. A lifestyle intervention is the first line treatment for CAD but has proven inadequate. Pharmacologic agents that inhibit 3-hydroxy-3-methylglutaryl coenzyme A (HMG-CoA) (statins) are being administered to inhibit cholesterol production. Other pharmacologic agents that can lower LDL and raised HDL are fibric acids, high-density lipoprotein stimulators (nicotinic acids), cholesterol absorption inhibitors (ezetimibe) and bile acid sequestrants. However, this pharmaceutics have important limitations, with only $38 \%$ of dyslipidemia and $18 \%$ of CAD patients attaining the National Cholesterol Education Program goals [2]. Statins, the fundamental therapy for reducing LDL levels, fail to allow majority of patients to their lipid goals. Therefore; additional therapeutic modalities to lower cholesterol levels needs to be explored.

In recent years there has been increasing interest in probiotics and several studies demonstrated cholesterol lowering effect of probiotics notably from Lactobacillus species. Some of its strains are proven to have cholesterol-lowering properties such as Lactobacillus casei, Lactobacillus fermentum, Lactobacillus delbrueckii [3] and Lactobacillus acidophilus [4]. There are several mechanisms proposed for the cholesterol lowering property of probiotics and had been described in a recent review. Lactobacillus bacteria can produce ferulic acid which can inhibit hepatic HMG-CoA reductase and promote the excretion of acidic sterol [5]. In addition, bile salt hydrolase activity of Lactobacillus has been reported [6]. On the other hand, bacteria have been reported to assimilate cholesterol [7]. Though the cholesterol-lowering properties of Lactobacillus brevis are still not well defined there have been extensive studies both in vivo and in vitro often yielding inconsistent results.

In view of the foregoing, the researchers have conceptualized to investigate Lactobacillus brevis cholesterol lowering effect through in-vitro experiments using bacterial media. This work may provide grounds for future investigations to select probiotic as a cholesterol-lowering therapeutic. 


\section{Materials AND Methods}

\subsection{Bacterial Growth Media and Cholesterol}

The De Man-Rogosa- Sharpe (MRS) agar and broth were purchased from DKL Laboratory Supplies (Manila, Philippines) and prepared according to manufacturer's instruction and stored at $4{ }^{\circ} \mathrm{C}$ until used. Water soluble cholesterol powder was also purchased from DKL Laboratory Supplies and was prepared according to manufacturer's instruction.

\subsection{Bacterial Strain}

The bacteria Lactobacillus brevis was purchased from the Philippine National Collection of Microorganisms at University of the Philippines- BIOTECH (Laguna, Philippines). The bacteria samples were stored at $4{ }^{\circ} \mathrm{C}$ and were streaked in MRS agar the following day and incubated at $37{ }^{\circ} \mathrm{C}$ with $5 \% \mathrm{CO} 2$.

\subsection{Bacterial Culture Conditions}

Prior to assay MRS agar plate was streaked with Lactobacillus brevis sample to ensure purity and incubated at $37^{\circ} \mathrm{C}$ with $5 \% \mathrm{CO} 2$ for $48 \mathrm{hrs}$. Colonies from the MRS agar were inoculated into $10 \mathrm{ml}$ MRS broth. The number of active-form bacteria inoculated in the MRS broth was adjusted to $3.0 \mathrm{x}$ $10^{5} \mathrm{CFU} / \mathrm{ml}$ by measuring the broth spectrophotometrically against $1 \mathrm{M} \mathrm{Mc}$ Farland Standard. Colony from the MRS agar plate was used to inoculate $3 \mathrm{ml}$ MRS broth.

\subsection{Determination of Cholesterol Assimilation in MRS broth}

The capability of Lactobacillus brevis to assimilate cholesterol was investigated in MRS broth. Increasing amount of water-soluble cholesterol powder $(10 \mathrm{mg} / 1,20 \mathrm{mg} / \mathrm{ml}, 40 \mathrm{mg} / \mathrm{ml}, 80 \mathrm{mg} / \mathrm{ml}$ and $160 \mathrm{mg} / \mathrm{ml}$ ) were added to each MRS broth with the bacterial inoculum. The control tubes were composed of $10 \mathrm{ml}$ sterile MRS broth with the same concentration of cholesterol as with the experimental tubes but without bacteria. The experimental and control tubes were incubated for 24 hours at $37^{\circ} \mathrm{C}$. Following incubation, viability was measured by standard colony counting methods. For cholesterol analysis, the incubated probiotic tubes were centrifuged at $4000 \mathrm{rpm}$ for 10 minutes in order to separate the bacterial biomass and obtain clear MRS broth supernatant containing nonassimilated cholesterol. The cholesterol concentration in the supernatant was assayed with cholesterol enzymatic diagnostic kit and read against sterile MRS broth blank at $546 \mathrm{~nm}$. The pellet was resuspended and mixed with distilled water and read against distilled water blank at $546 \mathrm{~nm}$. The experiments were performed in triplicates.

\section{ReSUlts}

\section{Cholesterol Concentration of the Control and Experimental Tubes}

The results that were gathered from the different bacterial suspensions of the control and the experimental were averaged. For the control, the average cholesterol concentration of the supernatant and pellet were 705.75 and $658.27 \mathrm{mg} / \mathrm{dl}$ respectively. For the experimental group, the average concentration of the supernatant was $359.06 \mathrm{mg} / \mathrm{dl}$ and 376.76 for the pellet. (Table 1) Statistical analysis using t-test was found to be significant at $95 \%$ significance level (Table 2.) This showed that the cholesterol concentration of the experimental group was lower than the control.

Table 1. Cholesterol concentration after $24 \mathrm{hr}$. incubation

\begin{tabular}{|c|c|c|c|c|}
\hline Parameter & \multicolumn{2}{|c|}{ Control $(\mathrm{mg} / \mathrm{dl})$} & \multicolumn{2}{c|}{ Experimental } \\
\hline & Supernatant & Pellet & Supernatant & Pellet \\
\hline Cholesterol concentration & 705.75 & 658.27 & 359.06 & 376.76 \\
\hline
\end{tabular}

Table 2. T-test result of supernatant and pellet

\begin{tabular}{|c|c|c|}
\hline & Supernatant & Pellet \\
\hline T-value & 52.814164 & 11.032325 \\
\hline p-value & $<0.00001$ & 0.000384 \\
\hline
\end{tabular}

Percent Cholesterol assimilation of Lactobacillus brevis

The percent cholesterol assimilation was computed using the formula: of Tomaro-Duchesneauet al. 2014: 


$$
\mathrm{C}=\frac{A-B}{A} \times 100
$$

where:

A - cholesterol concentration in MRS supernatant of the experimental group

B - cholesterol concentration in MRS supernatant of the control

C- \% cholesterol assimilation

The percent cholesterol assimilation of Lactobacillus was shown in Table 3. and showed highest assimilation in $10 \mathrm{mg}$ cholesterol concentration. The percent assimilation showed decreasing value as levels of concentration of cholesterol was increased in the MRS broth.

Table 3. Percent Cholesterol Assimilation

\begin{tabular}{|c|c|}
\hline Cholesterol Concentration & Percent Assimilation \\
\hline $10 \mathrm{mg}$ & 50.31 \\
\hline $20 \mathrm{mg}$ & 49.12 \\
\hline $40 \mathrm{mg}$ & 47.08 \\
\hline $80 \mathrm{mg}$ & 20.16 \\
\hline
\end{tabular}

\section{DisCUSSION}

Increased serum cholesterol concentration correlates highly with coronary arterial disease which is the leading cause of death worldwide. Probiotics have been investigated for its potential to lower cholesterol both in vitro and in-vivo. In the 1970's fermented milk containing a wild Lactobacillus strain was reported to have a hypocholesterolemic effect in humans [8]. Since then, many experiments have been conducted in vitro or in vivo to investigate the cholesterol-lowering effect of lactic acid bacteria (LAB), especially strains of Lactobacillus and Bifidobacterium [9] [10] [11].

The cholesterol reducing effect of probiotic bacteria have been attributed to its bile salt hydrolase activity [6] cholesterol assimilation and incorporation in cell membranes [12], bile deconjugation, and the production of compounds that can inhibit enzymes necessary for cholesterol production. Cholesterol assimilation by probiotic bacteria in the gastrointestinal tract would allow for the reduction of cholesterol absorption by enterocytes and excretion of the cholesterol from the host, as depicted in Table 3. This would, in turn, lead to a decreased risk of developing coronary artery disease.

The goal of the presented work was to investigate Lactobacillus brevis for its ability to assimilate cholesterol from bacterial culture media. Initially, MRS bacterial culture media was supplemented with varying concentrations of cholesterol and bacteria was added for $24 \mathrm{~h}$ of incubation. Cholesterol concentration was measured in supernatant and pellet by enzymatic colorimetric method after $24 \mathrm{~h}$ incubation. Lactobacillus brevis was shown to successfully lower cholesterol. There was significant reduction of cholesterol as compared with the negative control. The percent assimilation decreased as level of cholesterol increased and showed the highest.

Studies by previous groups have demonstrated cholesterol assimilation in the same range by Bifidobacterium longum subspecies infantis with $40 \mu \mathrm{g} / \mathrm{mL}$ and Bifidobacterium bifidum MB 109 assimilated $50 \mu \mathrm{g} / \mathrm{mL}$ of cholesterol in MRS broth [13]. Similarly, Lactobacillus plantarum could assimilate cholesterolintherangeof $14-22 \mu \mathrm{g} / \mathrm{mL}[14]$.

In addition, the hypocholesterolemic effect of probiotics in dairy products fermented with the appropriate strain(s) of bacteria induced a decrease in the level of circulating cholesterol concentrations. However, the strains found in fermented dairy products do not normally reside in the human intestinal tract [9]. Thus, daily consumption of probiotic products may be a dietary solution for inducing long-term hypocholesterolemic effects.

Future work may focus oninvestigationsintoothercholesterol-loweringproperties, including screening for bile salt hydrolase activity. In terms of cholesterol assimilation, the specific mechanism by which the cholesterol is removed from the supernatant should be determined. Ideally, a probiotic that would influence multiple targets, using bile salt hydrolase activity, reducing HMGCoA reductase activity, and assimilating cholesterol, would be developed. In addition, a probiotic formulation could be developed as a combination therapy with pharmaceutics such as statins. 


\section{Conclusion}

In our study, it was found that Lactobacillus brevis had significant cholesterol lowering effect in vitro because of its cholesterol assimilating property.

\section{RECOMMENDATION}

Further research is needed to determine the mechanism of cholesterol uptake and to determine whether or not ingestion of cells of a selected strain of L. acidophilus could decrease serum cholesterol levels in adult humans with primary hypercholesterolemia.

\section{REFERENCES}

[1] World Health Organization (2017, May). Fact Sheet: Cardiovascular diseases. Retrieved from http://www.who.int/mediacentre/factsheets/fs317/en/

[2] Pearson T., Laurora I., Chu H., Kafonek S. The Lipid Treatment Assessment Project (L-Tap): a multicenter survey to evaluate the percentages of dsylipidemic patients receiving lipid-lowering therapy and achieving low-density lipoprotein cholesterol goals. Arch Intern Med. 160:459-467 (200).

[3] Nguyen T., Kang J., Lee M. S. Characterization of Lactobacillus plantarum PH04, a potential probiotic bacterium with cholesterol-lowering effects. Int. J. Food Microbiol. 113:358-361 (2007)

[4] Fukushima, M., Yamada A., Endo T., Nakano M. Effects of a mixture of organisms, Lactobacillus acidophilus or streptococcus faecalis on D6-desaturase activity in the livers of rats fed a fat- and cholesterol-enriched diet. Nutrrients. 15: 373-378 (1999)

[5] Tomaro-Duchesneau C., Saha S., Malhotra M., Coussa-Charley M. Al-Salami, H., Jones, M., Labbe A., Prakash S. Lactobacillus fermentum NCIMB 5221 has a greater ferulic acid production compared to other ferulic acid esterase producing Lactobacilli. International Journal of Probiotics and Prebiotics, 7: 23-32 (2012)

[6] Jones M., Tomaro-Duchesneau C., Martoni C., Prakash S. Cholesterol lowering with bile salt hydrolase-active probiotic bacteria, mechanism of action, clinical evidence, and future direction for heart health applications. Expert Opinion on Biological Therapy: 13 (5), 631-642 (2013)

[7] Pereira D., Gibson G. Cholesterol assimilation by lactic acid bacteria and bifidobacteria isolated from the human gut. Applied and Environmental Microbiology: 68 (9), 4689-4693 (2002)

[8] Mann G., A. Spoerry A. Studies of a surfactant and cholesteremia in the Maasai, The American Journal of Clinical Nutrition, 27 (5), 464-469 (1974)

[9] Pan D., Zeng X., Yan T. Characterisation of Lactobacillus fermentum SM-7 isolated from koumiss, a potential probiotic bacterium with cholesterol-lowering effects. Journal of the Science of Food and Agriculture: 91 (3) 512-518 (2011)

[10] Oner O., Aslim B., Aydaş S. Mechanisms of cholesterol-lowering effects of lactobacilli and bifidobacteria strains as potential probiotics with their bsh gene analysis," Journal of Molecular Microbiology and Biotechnology: 24 (1), 12-18 (2014)

[11] Wang J., Zhang H.,Chen X., Chen Y., Bao Q. Selection of potential probiotic lactobacilli for cholesterol-lowering properties and their effect on cholesterol metabolism in rats fed a highlipid diet. Journal of Dairy Science: 95 (4) 1645-1654 (2012)

[12] Lye H., Rahmat-Ali G., LiongM. Mechanisms of cholesterol removal by lactobacilli under conditions that mimic the human gastrointestinal tract. International Dairy Journal: 20 (3), 169175(2010).

[13] Bordoni A., Amaretti A., Leonardi A., Boschetti E., Danesi F., MAtteuzi D., Roncaglia L., Raimondi S., Rossi M.Cholesterol-lowering probiotics: in vitro selection and in vivo testing of bifidobacteria. Applied Microbiology and Biotechnology: 97 (18), 8273-8281 (2013)

[14] Yu Z.,Zhang X.,Li S.,Li C., Li D., Yang Z., Evaluation of probiotic properties of Lactobacillus plantarum strains isolated from Chinese sauerkraut. World Journal of Microbiology and Biotechnology,29 (3) 489-498, (2013) 